\title{
Design and implementation of food traceability system based on two dimensional code
}

\author{
Li Tingting $^{1}$, Li Bo ${ }^{2}$, Huang Dechang ${ }^{2}$ \\ ${ }^{1}$ School of Software Engineering, East China Jiaotong University, Nanchang, 330013, PR China \\ ${ }^{2}$ School of Information Engineering, East China Jiaotong University, Nanchang, 330013, PR China
}

Email: libo_jiaoda@126.com

Keywords: Things agriculture; two dimensional code; food traceability; network optimization

\begin{abstract}
Food safety problem has become one of the most important topics of social concern, because of food from the acquisition of raw materials production to final consumption of intermediate links become more and more, traditional form filling, seal and other way is not easy to on these links were effective monitoring, the purpose of developing this system is to more effective and convenient management, monitoring of food from raw materials to the table the whole process, using the data of food industry chain integration, the construction of food safety traceability system, in a large extent can effectively ensure food safety, but also for major food safety problems, relevant departments back and verification provides a powerful technical support. This paper designed and developed of food safety traceability main software system modules; at the same time a large amount of data that need to be studied computing environment, software and hardware related technologies, the use of high performance computing model, distributed data processing model, a rapid, accurate, high performance data processing and analysis solutions.
\end{abstract}

\section{Introduction}

Food safety is directly related to people's health and life safety, as well as the national economic development and social harmony [1, 2]. In recent years, domestic food safety incidents frequently, from Sudan event to trimeric amine gas milk powder incident, to the gutter oil, to lean events and the poison capsule events, serious impact on the food security of domestic consumers. At the same time, our country's food is rejected by the exporting country, the detention, the return, the claim and the termination of the contract, the event has occurred, which has affected our country's economic development. Food safety events increasingly expanded, more and more by the concern of the government and people, government supervision is more and more strong [3-5].

Products in the library are given only the number of digital anti forgery, such as bar code. Two dimensional code security image, through two or more cameras on the product to obtain the anti false image, the acquired image is converted into digital signal, and then the 3D image information is transmitted to the computer for 3D image reconstruction, and the product is obtained. Identification of three-dimensional data, through the product information database to match the data, you can get the product details [6]. According to the requirements of the logistics information management system for the security identification image, a local matching algorithm is proposed, which can effectively distinguish the edge of the object [7].

With the two-dimensional code technology in more and more industries, products are widely used, the use of two-dimensional code technology into the food chain data, build food safety traceability system, in large part to effectively ensure food safety, while at the same time when major food safety problems, the relevant departments traceability, verification and other strong technical support offered. In this paper, the requirements of food traceability system, the use of software engineering methods, the system function, non functional requirements are analyzed, the system function, drawing a use case diagram, and design use case specification; food traceability system design, including system design, network topology design, according to the needs analysis database design. 


\section{QR code Data Processing and Services System}

Technical indicators dimensional code and QR code technology. QR code by the Japanese Denso Corporation is a two-dimensional matrix code symbols, it has a large information capacity. High reliability, may represent a variety of characters and images of text information [8]. A complete QR code generally consists of two-part graphic function and basic structure of the coding region .QR code is shown in Fig. 1.

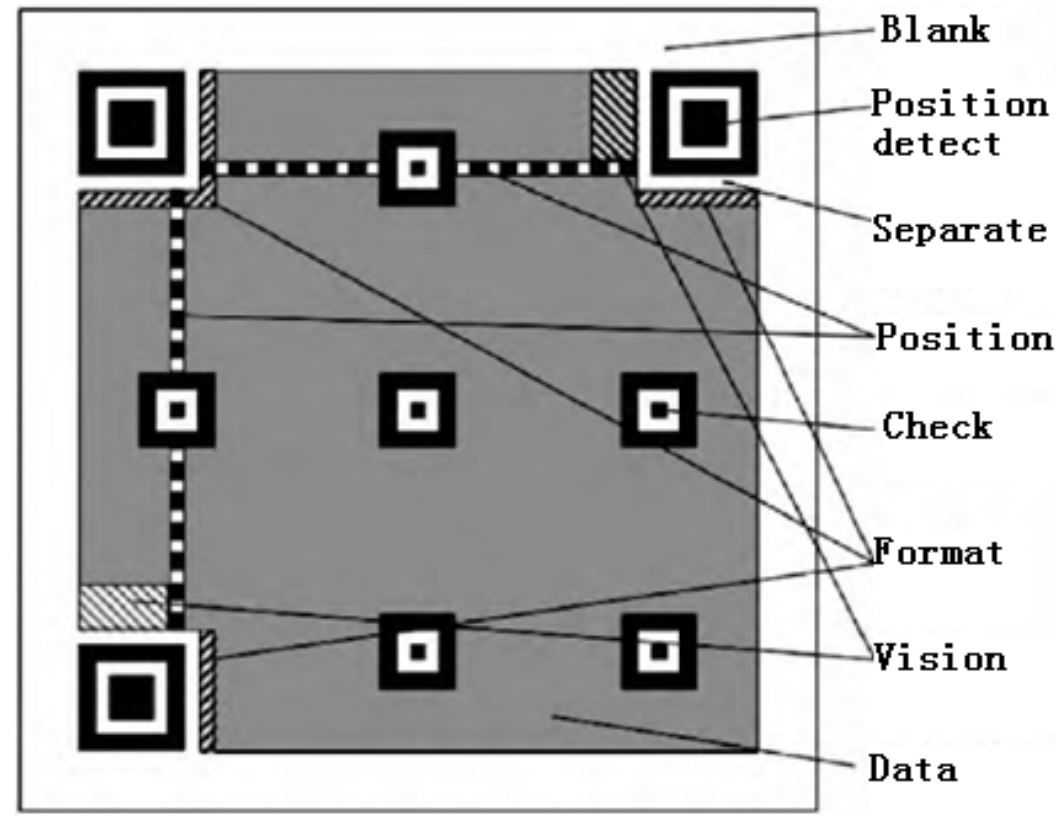

Fig. 1. Consist of QR code

Two-dimensional code main technical indicators is the information storage capacity and error correction information, two-dimensional code of unit area (per square inch) of information storage capacity, namely information density, it is the first important measure of our performance of two-dimensional code indicators, information density usually two-dimensional code is a one-dimensional bar code tens to hundreds of times, the two-dimensional code can not only prevent errors, but also to correct the mistake, even if partially damaged, but also can restore the information, which is one-dimensional code can not be compared.

Classification of two dimensional bar code. Two dimensional code is designed and developed on the basis of one dimension code (Fig. 2). The main design requirement of the two dimensional code is that it can store large amount of data and picture information. There are two main design ideas, one is based on the one-dimensional bar code, the other is to use the geometric structure and the image design of the two-dimensional bar code of encoding mechanism, the relevant theory of image recognition to achieve two-dimensional code. Matrix type two dimensional bar code is the use of matrix data arrangement, in the matrix corresponding to the location of the unit with black spots or other shapes to represent the "1", and the blank position that " 0 ", by the arrangement of the matrix to determine the matrix code. Matrix code is based on combined coding theory, and computer image processing technology based on a graphical symbols for automatic recognition of processing graphics code.
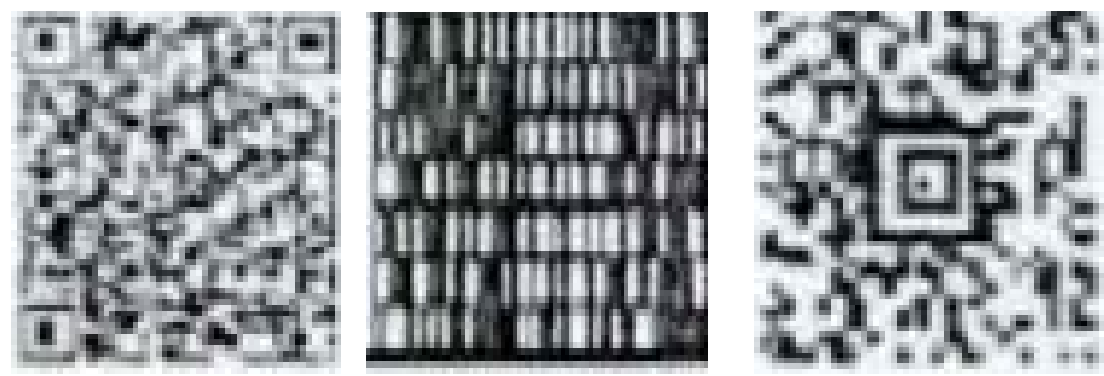

Fig. 2. Common two-dimensional code 


\section{Experimental results}

Application example model. Consumers or other system users in the use of the new system, the system may not be very familiar with the application interface, which requires that the user as long as the use of two-dimensional code scanning software scanning, you can immediately light and see the traceability data information, so the user interface is very important. For users to use the system may appear, to provide as much as possible to understand the online help, to guide users. Food traceability system in the collection of data information is very important, the system needs to provide a simple operation process, so that the system maintenance personnel to carry out data backup, verification and other daily management work, and when the system encountered a special event, the recovery of data information is also a measure of the maintenance of the important performance index system. Cotton category, for example, based on class - represents the policy was shown in Fig. 3 production rules knowledge - framework.

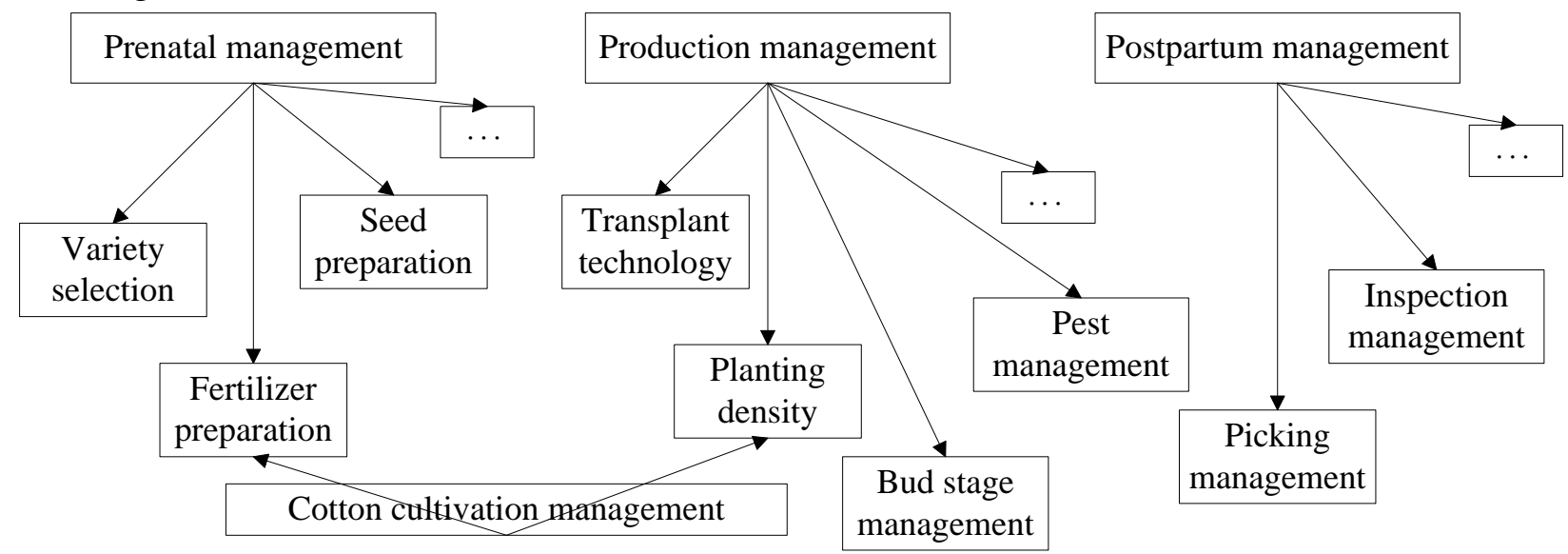

Fig. 3. The production rules knowledge-framework

The accuracy of the information: the system is to increase, delete, change the information provided by the production enterprise data, and it is to rely on authorization to achieve, so that the collection process of information to ensure that the monitoring of the accuracy of the data. Xinghai accurately reflected in system management personnel setting mechanism, the need for reconciliation processes, or managers of the misstatement, omission phenomenon occurred after, check personnel through the reconciliation of data information, discover and correct the mistakes and the safety of data security.

Fig. 4 is a method for predicting the present system of paddy moisture content of the sample and standard deviation values for dry moisture analyzer measured. Measured in 13 samples, sample bias $3,4,5$ absolute value between $1 \%$ to $2.3 \%$, and the remaining samples measuring deviations were less than $0.8 \%$, greater deviation measurement samples 3,4,5 reason Since the actual moisture within the rice grain bulk of these three samples is relatively high, balance and stability required for a long time, the longer the equilibrium predictions stabilization time closer to the true value.

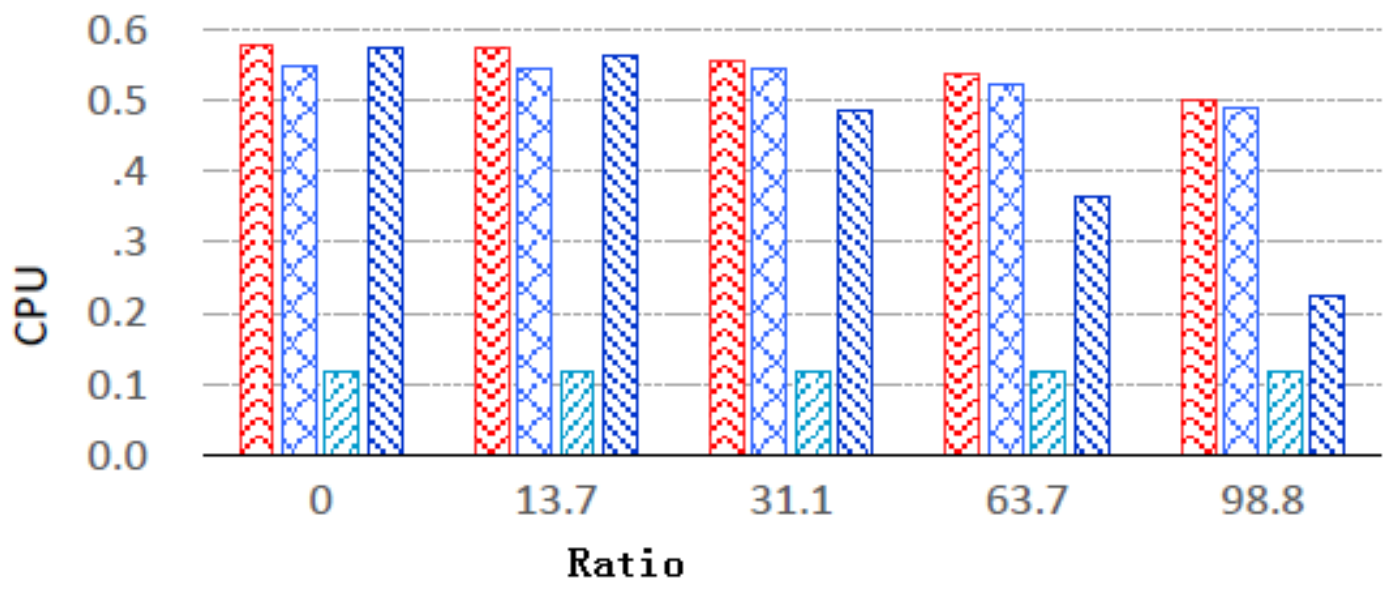

Fig. 4.The method for predicting the present system 
QR code management. QR code management module is an important foundation module system by food classified staff, the food industry batch administrator, system administrator QR code, consisting of four main parts to consumers. Which classified staff and administrators work batches corresponding to the system settings, select the appropriate food category and batches, so as to generate a QR code for each food used is QR code administrator in accordance with the information provided. QR code management using the example was shown in Fig. 5.

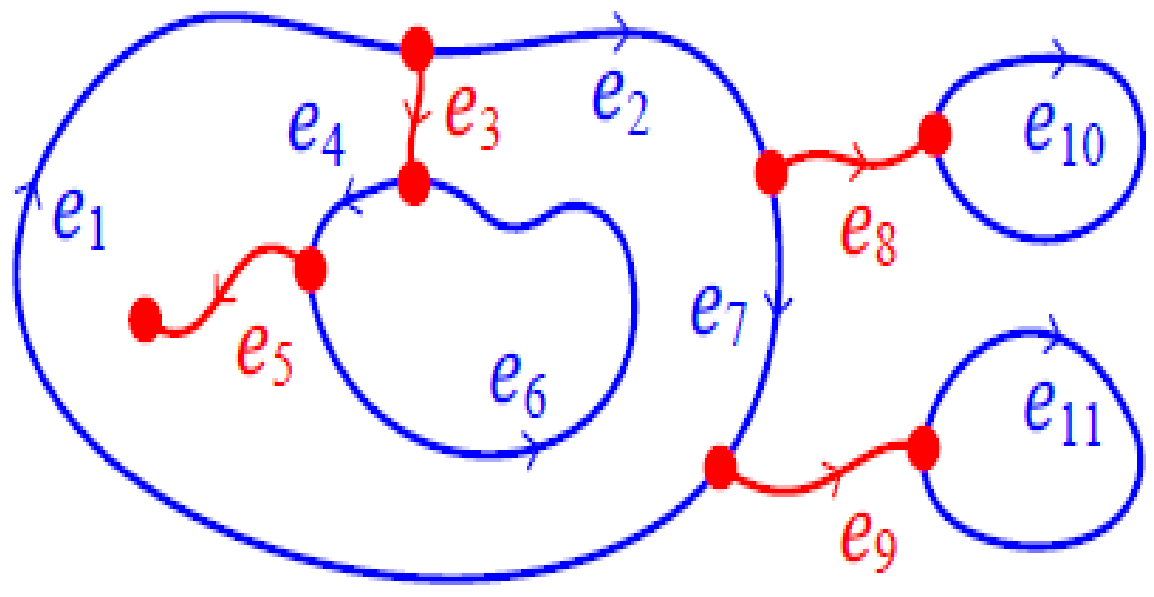

Fig. 5. QR code management

After the system according to the rules defined in the QR code generation, select classified information, batch information, click on the button, the system will Mr. into a 16 food code, converted a number of columns, and that generates a unique food box corresponding QR codes. The main participants in the system is the system administrator QR code, he classified according to the food business staff, information set by the administrator food companies generate batch of food boxes corresponding to the QR code, laying the foundation for food traceability, logistics and information query, the use case preconditions food business classification classified information who set the batch of food, food enterprises batch administrator settings of this batch of food information, post condition is to generate QR code and save it to the appropriate system database table.

Design of System Architecture. Technical architecture of the system was shown in Fig. 6, using the SSH framework, this 5-tier structure is reasonable definition, design and development work of the system is the main business logic design JSP page design, business layer presentation layer, database layer database design work. Among these, the mapping physical layer and persistence layer is automatically generated by development tools.

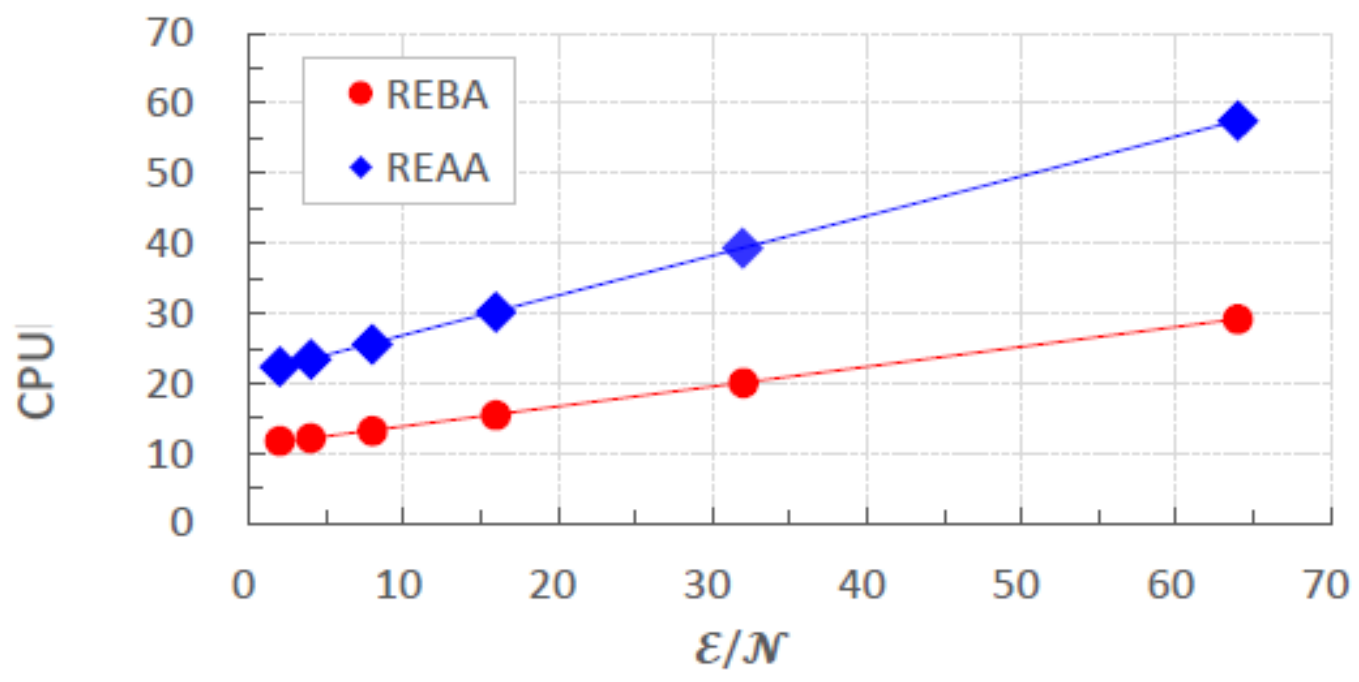

Fig. 6. Technical architecture of the system 


\section{Summary}

This paper designs and implements a food traceability system based on QR code according to the requirements of modern food traceability management. According to the actual situation of the project, the process of food production, packaging, logistics transportation and other aspects of the comprehensive analysis, design of the food traceability management program, the system uses QR code as the core carrier, application SSH framework, this paper focuses on the bar code generation subsystem, tracking subsystem, logistics information subsystem design, and intelligent mobile phone gradually widespread use, and make the system has good usability, timeliness, validity.

\section{References}

[1] F. Ma and F. Yang. The prediction of neural network to surface subsidence in mining. Chinese Journal of Geological Hazard and Control, 1995; 12(1),pp. 84-87.

[2] Tonkovic, Zlatko; Zekic-Susac, Marijana; Somolanji, Marija. Prediction of Natural Gas Consumption with Feed-forward and Fuzzy Neural Networks. Tehnicki Vjesnik-Technical Gazette. 3, 16(2009), pp. 51-61.

[3] Simunovic, Goran; Saric, Tomislav; Lujic, Roberto. Surface quality prediction by artificial-neural-networks. Tehnicki Vjesnik-Technical Gazette. 2, 16(2009), pp. 43-47.

[4] Elizabeth Chang, Tharam Dillon \& Farookh K. Hussain et al. Trust and Reputation for Service-oriented Environments: Technologies for Building Business Intelligenee and Consumer Confidence. England: John Wiley \& Sons, Ltd. 2006, 10(1):52.

[5] K.Itankura and K.Nakamura.A.public-key crptosysterm suitable for digital multisignatures.NEC J.Res.\& Dev 71.1983,pp.1-8.

[6] K. Ohta and T. Okamoto. Multisignature schemes secure against active insiderattacks. IEICE Trans. Fundamentals, E82-A(1), 1999, pp.1-31.

[7] T. Okamoto. A digital multisignature scheme using bijective public-key cryptosystems. ACM Trans. Computer Systems, 6(4), ACM Press, New York, 1988, pp.432-441.

[8] A. Boldyreva. Threshold signature, multisignature and blind signature schemes based on the gap-Diffie-Hellmangroup signature scheme. In Proceedings of PKC 2003, LNCS 2567 , Springer, Berlin, 2003, pp.31-46. 